\title{
Transcranial alternating current stimulation (tACS)
}

\section{Andrea Antal* and Walter Paulus}

Department of Clinical Neurophysiology, Georg-August University of Göttingen, Göttingen, Germany

\section{Edited by:}

Marom Bikson, The City University of New York, USA

\section{Reviewed by:}

Davide Reato, The City University of New York, USA

Michael A. Hunter, The University

of New Mexico, USA

*Correspondence:

Andrea Antal, Department of Clinical Neurophysiology, University Medical

Center, Georg-August University of Göttingen, Robert Koch Straße 40,

37075 Göttingen, Germany

e-mail: aantal@gwdg.de
Transcranial alternating current stimulation (tACS) seems likely to open a new era of the field of noninvasive electrical stimulation of the human brain by directly interfering with cortical rhythms. It is expected to synchronize (by one single resonance frequency) or desynchronize (e.g., by the application of several frequencies) cortical oscillations. If applied long enough it may cause neuroplastic effects. In the theta range it may improve cognition when applied in phase. Alpha rhythms could improve motor performance, whereas beta intrusion may deteriorate them. TACS with both alpha and beta frequencies has a high likelihood to induce retinal phosphenes. Gamma intrusion can possibly interfere with attention. Stimulation in the "ripple" range induces intensity dependent inhibition or excitation in the motor cortex (M1) most likely by entrainment of neuronal networks, whereas stimulation in the low $\mathrm{kHz}$ range induces excitation by neuronal membrane interference. TACS in the $200 \mathrm{kHz}$ range may have a potential in oncology.

Keywords: tACS, oscillations, human brain, motor, visual

\section{INTRODUCTION}

Transcranial alternating current stimulation (tACS) - the external application of oscillating electrical currents-is able to influence cortical excitability and activity (Antal et al., 2008; Chaieb et al., 2011; Moliadze et al., 2012; Wach et al., 2013). With exceptions (Marshall et al., 2006; Neuling et al., 2012) tACS is applied in most studies without a DC offset. It's simple form uses sinusoidal stimulation; however, any other waveform appears possible, such as rectangular current shapes. Ten $\mathrm{Hz}$ tACS was modeled in a realistic head model and was suggested to generate larger and more focused fields than DC stimulation (Manoli et al., 2012); with applied frequencies of 100 up to $1000 \mathrm{~Hz}$ a decrease of the size of this electrical field was assumed. This claim was, however, only based on differences in skin resistance at low frequencies which renders the statement quite doubtful (Paulus and Opitz, 2013). The major parameters that can shape the direction and the duration of the tACS-induced effects are the frequency, the intensity and the phase of the stimulation. The effect of duration of tACS on motor evoked potential (MEP) has not been systematically investigated yet. Increasing the duration of transcranial direct current stimulation (tDCS) results in a prolongation of the induced aftereffects (Nitsche and Paulus, 2000) up to about $13 \mathrm{~min}$ whereas doubling the 13 stimulation to $26 \mathrm{~min}$ inverses MEP aftereffects into inhibition (Batsikadze et al., 2013) It is unclear if this can be translated to tACS, too.

\section{ACS EFFECTS IN THE NORMAL BRAIN FREOUENCY OF THE STIMULATION}

tACS may be applied in a wide frequency range. At present data are available between close to DC up to $5 \mathrm{kHz}$ for plasticity studies (Chaieb et al., 2011) and $200 \mathrm{kHz}$ for tumor therapy (Kirson et al., 2007) using a single frequency. However, any combination of frequencies is possible: one special form of tACS is transcranial random noise stimulation (tRNS), which so far has been studied with a frequency spectrum between $0.1 \mathrm{~Hz}$ and $640 \mathrm{~Hz}$ with a "white noise" characteristic (Terney et al., 2008).

tACS applied at conventional EEG frequencies $(0.1-80 \mathrm{~Hz})$ and in the so called "ripple" range $(140 \mathrm{~Hz}$, see below) (Moliadze et al., 2010) may be able to interact with ongoing rhythms in the cortex. A very low frequency $(0.75 \mathrm{~Hz})$ stimulation combined with DC offset during non-rapid-eye-movement sleep in healthy humans enhances the retention of hippocampusdependent declarative memories when tested the next morning (Marshall et al., 2006). The DC offset used in this study leaves open the possibility of a DC effect (Bergmann et al., 2009).

Effects of tACS applied in the EEG range might differ depending on the outread parameters. A trend toward MEP inhibition following $10 \mathrm{~Hz} \mathrm{AC}$ stimulation over the primary motor cortex (M1) was observed (Antal et al., 2008), while $10 \mathrm{~Hz}$ stimulation improved visuomotor implicit learning, using a serial reaction time task. In the MEP measurement shorter stimulation duration (5 min) was applied whereas tACS in the implicit learning study lasted about twice as long. Nevertheless, a dissociation between MEP excitability changes and implicit learning under tACS has already been described (Moliadze et al., 2010). $140 \mathrm{~Hz}$ stimulation induces the largest MEP increase, whereas only $250 \mathrm{~Hz}$ improved implicit motor learning.

In another study, whereas $20 \mathrm{~Hz}$ tACS over the M1 increased corticospinal excitability (Feurra et al., 2011) as measured by MEP size, it slowed down voluntary movements using a visuomotor task (Pogosyan et al., 2009) but in parallel it increased beta coherence between scalp-recorded activity and electromyographic activity (EMG) of the first dorsal interosseus muscle. Opposing effects at beta and gamma frequencies depending on phase of a of motor task exist (Joundi et al., 2012): using a visually driven go-no-go task stimulation at $20 \mathrm{~Hz}$ afforded a significant but modest slowing of force production in the go task, however, stimulation in no-go trials, where the triggered motor task 
involved inhibition, led to a major reduction in force generation. In contrast, $70 \mathrm{~Hz}$ tACS was ineffective during errors of commission following no-go cues, but increased performance during go trials.

TACS up to $80 \mathrm{~Hz}$ elicits phosphenes in a frequency- and intensity dependent way (Turi et al., 2013). When applied over the occipital cortex, the perception of phosphenes was peaking at about $15 \mathrm{~Hz}$ in brightness with a lower peak in darkness (Kanai et al., 2008). Although electrodes were placed over $\mathrm{Oz}$ and $\mathrm{Cz}$, this effect was probably induced by far field stimulation at the retina (Schutter and Hortensius, 2010). TACS can probably only influence visual cortical functions at a subthreshold level as shown by modification of transcranial magnetic stimulation (TMS)induced phosphene-thresholds (Kanai et al., 2010). Furthermore, contrast-discrimination thresholds were decreased only during $60 \mathrm{~Hz}$ tACS, but not during 40 and $80 \mathrm{~Hz}$ stimulations (Laczo et al., 2012).

TACS applied over the PO9 and PO10 EEG electrode positions at the individual alpha frequency range $(8-12 \mathrm{~Hz})$ induced an entrainment of the applied oscillatory activity (Zaehle et al., 2010). However, it was recently documented that the after-effects of tACS applied at the individual alpha frequency may depend on the individual endogenous power: tACS was effective only under conditions of low endogenous alpha frequency power (Neuling et al., 2013). Furthermore, when stimulation frequency was fixed at 6 and $10 \mathrm{~Hz}$, tACS impaired performance in the visual detection task (Brignani et al., 2013).

tACS applied outside the conventional EEG frequency range, e.g., with frequencies of $140 \mathrm{~Hz}$ and in the low $\mathrm{kHz}$ range $(1-5 \mathrm{kHz})$ increases excitability in a similar way than anodal tDCS, when $1 \mathrm{~mA}$ intensity is used (Moliadze et al., 2010; Chaieb et al., 2011). Stimulation at $80 \mathrm{~Hz}$ remains without an effect, while $250 \mathrm{~Hz}$ clearly had a delayed onset and shorter lasting response, compared to the MEP increase observed during and after $140 \mathrm{~Hz}$ tACS.

The tRNS paradigm was developed with a potential to desynchronize normal and pathological cortical rhythms (Terney et al., 2008). The rationale behind this method is a possible entrainment with cortical oscillations of different frequencies at the same time. This may apply for intra-areal with higher oscillation frequencies or for inter-areal oscillations with lower frequencies. Input noise plays a role in sensitizing neuronal systems through a mechanism known as stochastic resonance (Wiesenfeld and Moss, 1995). Alternatively, impaired signal detection might be improved by input noise in order to sensitise sensory processing (Moss et al., 2004). An excitability increase lasting up to $90 \mathrm{~min}$, observed both for MEP measures and behavioral tasks, was induced after $10 \mathrm{~min}$ of tRNS. Unexpectedly higher frequencies $(100-640 \mathrm{~Hz})$ and not frequencies less than $100 \mathrm{~Hz}$ were responsible for this excitability increase.

The efficacy of the stimulation seems to be dependent on the type of the task and on the power of intrinsic oscillations at baseline (Neuling et al., 2013) and the involvement of the different memory systems in a given cognitive task: when tRNS was applied over the dorsolateral prefontal cortex (DLPFC) subjects made more mistakes in a probabilistic classification task (Ambrus et al., 2011), whereas when using the n-back task no significant change in performance was found (Mulquiney et al., 2011). When tRNS was applied of the visual cortex improved neuroplasticity in a perceptual learning paradigm (Fertonani et al., 2011). Nevertheless, the neuronal mechanisms underlying the effect of tRNS might be different from those of tACS, using a single stimulation frequency.

\section{INTENSITY OF THE STIMULATION}

The effect of tACS appears to be intensity dependent. A trend in a first study (Antal et al., 2008) using a low intensity of $0.4 \mathrm{~mA}$ over the M1 toward MEP inhibition following $10 \mathrm{~Hz}$ AC stimulation was confirmed later with higher frequencies (Moliadze et al., 2012). Other tACS frequencies between 5 and $40 \mathrm{~Hz}$ failed to induce any measurable aftereffects at this (too) low intensity.

Interestingly, both tACS at $140 \mathrm{~Hz}$ and tRNS show an intensity-dependent aftereffect: whereas $0.2 \mathrm{~mA}$ intensity has no effect an intensity of $0.4 \mathrm{~mA}$ leads to inhibition, 0.6 and $0.8 \mathrm{~mA}$ do not provide a significant effect (Moliadze et al., 2012). With $1 \mathrm{~mA}$ an increase of the MEP amplitudes can be seen. This suggests that inhibitory circuits can be excited preferentially with lower intensities, an effect which has also been documented for TMS (Berger et al., 2011). Nevertheless, the reason for this observed reversal in the direction of MEP effects induced by higher frequency stimulation at different intensities has not been clarified yet. It is likely that $140 \mathrm{~Hz}$ and tRNS at the lower intensity only facilitate intracortical inhibitory networks of corticospinal motoneurons, thus resulting in net inhibition of MEP amplitudes (Pashut et al., 2011). It also cannot be excluded that stimulation applied at $0.4 \mathrm{~mA}$ may inhibit intracortical facilitatory effects on corticospinal motoneurons. When recording in a pyramidal neuron located in layer 5 of the rat cortex the composite response to an electrical stimulation of various layers $(2-3,4$, or 6$)$, in terms of excitationinhibition balance, resulted in conductance changes consisting of $20 \%$ excitation and $80 \%$ inhibition, independent from the stimulated layer. Moreover, it was shown that excitatory circuits are strongly controlled by inhibitory circuits (Maffei et al., 2004) by feedback and feed-forward connections (Bannister, 2005).

\section{PHASE OF THE STIMULATION}

Brain oscillations are characterized in addition to frequency and power by their phase. Modeling studies propose that in active neuronal networks weak electrical fields can induce small but coherent changes in the firing rate and timing of neuronal populations that can be magnified by dynamic network activity (Radman et al., 2007; Reato et al., 2010) When stimulating the left frontal and parietal cortex by $6 \mathrm{~Hz}$ tACS in phase, cognitive performance in a delayed letter discrimination task was improved, when stimulating out of phase it was delayed (Polania et al., 2012). In a recent study stimulating the temporal cortex using $10 \mathrm{~Hz}$ with DC-offset it was found that manipulation of the phase resulted in different auditory detection thresholds, which supports the notion that perception can be periodically modulated by oscillatory processes (Neuling et al., 2012). Nevertheless, the DC offset used in this study leaves open the possibility of a DC effect. 


\section{MECHANISMS OF ACTION}

tACS applied in the EEG range is believed to mainly entrain with or synchronize neuronal networks, thus inducing changes in ongoing oscillatory brain activity. Indeed, spike synchrony of converging input has been shown to enhance the information transfer and speed up processing (e.g., Butts et al., 2007). Nevertheless, stimulation applied in the $\mathrm{kHz}$ range probably does not interfere with oscillatory activity, but targets the membrane excitability of neurons more selectively. It could be that the temporary modification of the synapse once exposed to a rapidly alternating electrical field, alters the associated biochemical mechanisms, such as accumulation of calcium in the presynaptic nerve terminals leading to short-term synaptic plasticity effects (Citri and Malenka, 2008).

The mechanisms of tRNS so far are unclear, if e.g., repeated opening of $\mathrm{Na}^{+}$channels or a higher sensitivity of neuronal networks to electrical field modulation than the single neuron threshold (Francis et al., 2003).

\section{APPLICATIONS IN DISEASE}

tACS would have a particular indication in disorders in which abnormal oscillatory patterns may play a role, such as Parkinson's disease or schizophrenia (Gonzalez-Burgos and Lewis, 2008; Burns et al., 2011) by attenuating or resetting anomalous oscillations. Indeed, Parkinsonian resting tremor could be bisected by tACS of the M1 at specified phase alignments (Brittain et al., 2013).

Using $200 \mathrm{kHz}$ frequency a pilot clinical trial was carried out treating human patients suffering from recurrent gliobastoma (Kirson et al., 2007). By transcranial application of continuous high frequency stimulation inhibits the growth of this treatmentresistant tumor, with little or no side effects, pursuing the concept that dividing tumor cells can be destroyed during mitosis.

\section{REFERENCES}

Ambrus, G. G., Paulus, W., and Antal, A. (2010). Cutaneous perception thresholds of electrical stimulation methods: comparison of tDCS and tRNS. Clin. Neurophysiol. 121, 1908-1914. doi: 10.1016/j.clinph.2010.04.020

Ambrus, G. G., Zimmer, M., Kincses, Z. T., Harza, I., Kovacs, G., Paulus, W., et al. (2011). The enhancement of cortical excitability over the DLPFC before and during training impairs categorization in the prototype distortion task. Neuropsychologia 49, 1974-1980.

Antal, A., Boros, K., Poreisz, C., Chaieb, L., Terney, D., and Paulus, W. (2008). Comparatively weak after-effects of transcranial alternating current stimulation (tACS) on cortical excitability in humans. Brain Stimul. 1, 97-105. doi: 10.1016/j.brs.2007.10.001

Bannister, A. P. (2005). Inter- and intralaminar connections of pyramidal cells in the neocortex. Neurosci. Res. 53, 95-103.
Batsikadze, G., Moliadze, V., Paulus, W., Kuo, M. F., and Nitsche, M. A. (2013). Partially non-linear stimulation intensity-dependent effects of direct current stimulation on motor cortex excitability in humans. J. Physiol. 591, 1987-2000.

Berger, U., Korngreen, A., BarGad, I., Friedman, A., Wolfus, S., Yeshurun, Y., et al. (2011). Magnetic stimulation intensity modulates motor inhibition. Neurosci. Lett. 504, 93-97. doi: 10.1016/j.neulet.2011.09.004

Bergmann, T. O., Groppa, S., Seeger, M., Molle, M., Marshall, L., and Siebner, H. R. (2009). Acute changes in motor cortical excitability during slow oscillatory and constant anodal transcranial direct current stimulation. J. Neurophysiol. 102, 2303-2311. doi: 10.1152/jn.00437.2009

Brignani, D., Ruzzoli, M., Mauri, P., and Miniussi, C. (2013). Is transcranial alternating current stimulation effective in modulating brain

Applying the current transorbitally at the individual phosphene thresholds ACS is effective in the therapy following optic nerve injury in human (Gall et al., 2010; Sabel et al., 2011).

\section{COMPARISON TO rTMS?}

Both rTMS and tACS could provide the basis to interact with or induce local, probably also remote oscillatory activity. While rTMS involves delivering a brief, repetitive, high-intensity magnetic pulses to the head through a coil that induces electrical currents in a focal area underneath this area, with regard to tACS, oscillatory current is delivered with a battery-driven stimulator by means of a large electrode located on the area of interest and a reference electrode that is placed over a neutral area. tACS has some advantages compared to rTMS: (1) it is clearly cheaper due to the small and compact equipment; (2) it can be more easily combined with online cognitive projects; (3) it produces no acoustic noise and muscle twitching of cranial muscles and it causes much less or no perceptual skin sensations (Ambrus et al., 2010; Turi et al., 2013) and is hereby more suitable for double-blind, sham-controlled studies. Nevertheless, there are disadvantages, including shunting of the electric currents through the scalp and the skin irritations that sometimes can be observed under electrodes.

\section{FUTURE DIRECTIONS}

tACS is only in its beginnings. A seemly indefinitely number of stimulation paradigms will have to be condensed to those with highest physiological relevance. As a prerequisite, this requires a clearer picture of the neuronal mechanisms involved in tACSinduced entrainment. Knowledge of their dynamics over time would enable to formulate optimized protocols for future tACS studies.

oscillations. PLOS ONE 8:e56589. doi: 10.1371/journal.pone.0056589

Brittain, J. S., Probert-Smith, P., Aziz, T. Z., and Brown, P. (2013). Tremor suppression by rhythmic transcranial current stimulation. Curr. Biol. 23, 436-440. doi: 10.1016/j.cub.2013.01.068

Burns, S. P., Xing, D., and Shapley, R. M. (2011). Is gamma-band activity in the local field potential of V1 cortex a "clock" or filtered noise. J. Neurosci. 31, 9658-9664.

Butts, D. A., Weng, C., Jin, J., Yeh, C. I., Lesica, N. A., Alonso, J. M., et al. (2007). Temporal precision in the neural code and the timescales of natural vision. Nature 449, 92-95. doi: 10.1038/nature06105

Chaieb, L., Antal, A., and Paulus, W. (2011). Transcranial alternating current stimulation in the low $\mathrm{kHz}$ range increases motor cortex excitability. Restor. Neurol. Neurosci 29, 167-175.

Citri, A., and Malenka, R. C. (2008). Synaptic plasticity: multiple forms, functions, and mechanisms.
Neuropsychopharmacology 33, 18-41. doi: 10.1038/sj.npp.1301559 Fertonani, A., Pirulli, C., and Miniussi, C. (2011). Random noise stimulation improves neuroplasticity in perceptual learning. J. Neurosci. 31, 15416-15423. doi: 10.1523/JNEUROSCI.2002-11.2011

Feurra, M., Paulus, W., Walsh, V., and Kanai, R. (2011). Frequency specific modulation of human somatosensory cortex. Front. Psychol. 2:13. doi: 10.3389/fpsyg.2011.00013

Francis, J. T., Gluckman, B. J., and Schiff, S. J. (2003). Sensitivity of neurons to weak electric fields. J. Neurosci. 23, 7255-7261.

Gall, C., Fedorov, A. B., Ernst, L., Borrmann, A., and Sabel, B. A (2010). Repetitive transorbital alternating current stimulation in optic neuropathy. NeuroRehabilitation 27, 335-341.

Gonzalez-Burgos, G., and Lewis, D. A. (2008). GABA neurons and the mechanisms of network oscillations: implications for understanding cortical 
dysfunction in schizophrenia. Schizophr. Bull. 34, 944-961. doi: 10.1093/schbul/sbn070

Joundi, R. A., Jenkinson, N., Brittain, J. S., Aziz, T. Z., and Brown, P. (2012). Driving oscillatory activity in the human cortex enhances motor performance. Curr. Biol. 22, 403-407. doi: 10.1016/j.cub.2012.01.024

Kanai, R., Chaieb, L., Antal, A., Walsh, V., and Paulus, W. (2008). Frequency-dependent electrical stimulation of the visual cortex. Curr. Biol. 18, 1839-1843.

Kanai, R., Paulus, W., and Walsh, V. (2010). Transcranial alternating current stimulation (tACS) modulates cortical excitability as assessed by TMS-induced phosphene thresholds. Clin. Neurophysiol. 121, 1551-1554. doi: 10.1016/j.clinph.2010.03.022

Kirson, E. D., Dbaly, V., Tovarys, F., Vymazal, J., Soustiel, J. F., Itzhaki, A., et al. (2007). Alternating electric fields arrest cell proliferation in animal tumor models and human brain tumors. Proc. Natl. Acad. Sci. U.S.A. 104, 10152-10157. doi: 10.1073/pnas.0702916104

Laczo, B., Antal, A., Niebergall, R., Treue, S., and Paulus, W. (2012). Transcranial alternating stimulation in a high gamma frequency range applied over V1 improves contrast perception but does not modulate spatial attention. Brain Stimul. 5, 484-491. doi: 10.1016/j.brs.2011. 08.008

Maffei, A., Nelson, S. B., and Turrigiano, G. G. (2004). Selective reconfiguration of layer 4 visual cortical circuitry by visual deprivation. Nat. Neurosci. 7, 1353-1359.

Manoli, Z., Grossman, N., and Samaras, T. (2012). Theoretical investigation of transcranial alternating current stimulation using realistic head model. Conf. Proc. IEEE Eng. Med. Biol. Soc. 2012, 4156-4159. doi: 10.1109/EMBC.2012.6346882

Marshall, L., Helgadottir, H., Molle, M., and Born, J. (2006). Boosting slow oscillations during sleep potentiates memory. Nature 444, 610-613. doi: 10.1038 /nature 05278

Moliadze, V., Antal, A., and Paulus, W. (2010). Boosting brain excitability by transcranial high frequency stimulation in the ripple range. J. Physiol. 588, 4891-4904.

Moliadze, V., Atalay, D., Antal, A. and Paulus, W. (2012). Close to threshold transcranial electrical stimulation preferentially activates inhibitory networks before switching to excitation with higher intensities. Brain Stimul. 5, 505-511. doi: 10.1016/j.brs.2011.11.004

Moss, F., Ward, L. M., and Sannita, W. G. (2004). Stochastic resonance and sensory information processing: a tutorial and review of application. Clin. Neurophysiol. 115, 267-281. doi: 10.1016/j.clinph.2003.09.014

Mulquiney, P. G., Hoy, K. E., Daskalakis, Z. J., and Fitzgerald, P. B. (2011). Improving working memory: exploring the effect of transcranial random noise stimulation and transcranial direct current stimulation on the dorsolateral prefrontal cortex. Clin. Neurophysiol. 122, 2384-2389. doi: 10.1016/j.clinph.2011.05.009

Neuling, T., Rach, S., and Herrmann, C. S. (2013). Orchestrating neuronal networks: sustained after-effects of transcranial alternating current stimulation depend upon brain states. Front. Hum. Neurosci. 7:161. doi: 10.3389/fnhum.2013.00161

Neuling, T., Rach, S., Wagner, S., Wolters, C. H., and Herrmann, C. S. (2012). Good vibrations: oscillatory phase shapes perception. Neuroimage 63, 771-778. doi: 10.1016/j.neuroimage.2012.07.024

Nitsche, M. A., and Paulus, W. (2000). Excitability changes induced in the human motor cortex by weak transcranial direct current stimulation. J. Physiol. 527( $\mathrm{Pt} 3)$, 633-639.

Pashut, T., Wolfus, S., Friedman, A., Lavidor, M., Bar-Gad, I., Yeshurun, Y., et al. (2011). Mechanisms of magnetic stimulation of central nervous system neurons. PLoS Comput. Biol. 7:e1002022. doi: 10.1371/journal.pcbi.1002022

Paulus, W., and Opitz, A. (2013). Ohm's law and tDCS over the centuries. Clin. Neurophysiol. 124, 429-430. doi: 10.1016/j.clinph.2012.08.019

Pogosyan, A., Gaynor, L. D., Eusebio, A., and Brown, P. (2009). Boosting cortical activity at Beta-band frequencies slows movement in humans. Curr. Biol. 19, 1637-1641.

Polania, R., Nitsche, M. A., Korman, C., Batsikadze, G., and Paulus, W. (2012). The importance of timing in segregated theta phase-coupling for cognitive performance. Curr. Biol. 22, 1314-1318.

Radman, T., Su, Y., An, J. H., Parra L. C., and Bikson, M. (2007). Spike timing amplifies the effect of electric fields on neurons: implications for endogenous field effects. J. Neurosci. 27, 3030-3036.

Reato, D., Rahman, A., Bikson, M., and Parra, L. C. (2010). Low-intensity electrical stimulation affects network dynamics by modulating population rate and spike timing. J. Neurosci. 30, 15067-15079. doi: 10.1523/JNEUROSCI.2059-10.2010

Sabel, B. A., Fedorov, A. B., Naue, N., Borrmann, A., Herrmann, C. and Gall, C. (2011). Non-invasive alternating current stimulation improves vision in optic neuropathy. Restor. Neurol. Neurosci. 29, 493-505.

Schutter, D. J., and Hortensius, R. (2010). Retinal origin of phosphenes to transcranial alternating current stimulation. Clin. Neurophysiol. 121, 1080-1084. doi: 10.1016/j.clinph.2009.10.038

Terney, D., Chaieb, L., Moliadze, V., Antal, A., and Paulus, W. (2008). Increasing human brain excitability by transcranial high-frequency random noise stimulation. J. Neurosci. 28, 14147-14155. doi: 10.1523/JNEUROSCI.4248-08.2008

Turi, Z., Ambrus, G. G., Janacsek, K., Emmert, K., Hahn, L., Paulus,
W., et al. (2013). Both the cutaneous sensation and phosphene perception are modulated in a frequency-specific manner during transcranial alternating current stimulation. Restor. Neurol. Neurosci. 31, 275-285. doi: 10.3233/RNN-120297

Wach, C., Krause, V., Moliadze, V. Paulus, W., Schnitzler, A., and Pollok, B. (2013). Effects of $10 \mathrm{~Hz}$ and $20 \mathrm{~Hz}$ transcranial alternating current stimulation (tACS) on motor functions and motor cortical excitability. Behav. Brain Res. 241, 1-6. doi: 10.1016/j.bbr. 2012.11.038

Wiesenfeld, K., and Moss, F. (1995). Stochastic resonance and the benefits of noise: from ice ages to crayfish and SQUIDs. Nature 373, 33-36. doi: $10.1038 / 373033 \mathrm{a} 0$

Zaehle, T., Rach, S., and Herrmann, C. S. (2010). Transcranial alternating current stimulation enhances individual alpha activity in human EEG. PLOS ONE 5:e13766. doi: 10.1371/journal.pone.0013766

Conflict of Interest Statement: The authors declare that the research was conducted in the absence of any commercial or financial relationships that could be construed as a potential conflict of interest.

Received: 30 April 2013; accepted: 10 June 2013; published online: 28 June 2013.

Citation: Antal A and Paulus W (2013) Transcranial alternating current stimulation (tACS). Front. Hum. Neurosci. 7:317. doi: 10.3389/fnhum.2013.00317 Copyright (C) 2013 Antal and Paulus. This is an open-access article distributed under the terms of the Creative Commons Attribution License, which permits use, distribution and reproduction in other forums, provided the original authors and source are credited and subject to any copyright notices concerning any third-party graphics etc. 